\title{
Guía para un Plan de egreso en personas con secuelas por Enfermedad Vascular Cerebral
}

\author{
Discharge plan guides for persons with \\ Cerebrovascular Disease Sequelae
}

\section{Guia para um Plano de egresso em pessoas com sequelas por Doenças Vasculares Cerebrais}

\author{
A. Diaz-Ávila ${ }^{\mathrm{a}}$, C. Intriago-Ruíz ${ }^{\mathrm{b}}$ \\ ORCID: \\ ${ }^{\mathrm{a}} \mathrm{OOOOO-0003-3988-5937}$ \\ boooo-0003-0037-6086
}

Especialidad en Enfermería de Atención en el Hogar, División de Estudios de Posgrado, Escuela Nacional de Enfermería y Obstetricia, Universidad Nacional Autónoma de México, Ciudad de México, México

Recibido: 23 abril 2018/Aceptado: 22 noviembre 2018

\section{RESUMEN}

Introducción: Las personas que sufren un Evento Vascular Cerebral (EVC), generalmente, quedan incapacitadas para la realización de sus actividades básicas, lo que conlleva a una dependencia. Por consiguiente, es importante la elaboración de un plan de egreso con amplia información sobre los cuidados que facilite al profesional de enfermería brindar educación para la salud, dirigida a familiares y enfermos sobre el cuidado en el hogar tras un EVC. Métodos: El procedimiento utilizado para esta propuesta constó de tres fases, en las cuales se realizó una revisión de la literatura, una delimitación con base en el tema central, para finalmente, elaborar el plan de egreso que aquí se presenta.

Resultados: En la primera fase se analizaron 39 estudios, 16 de tipo cualitativo, 11 cuantitativos, ocho Guías de práctica clínica, tres mixtos y dos tesis; posteriormente, en la segunda fase se estableció la delimitación del tema con base en el criterio de evidencia científica y los 
diagnósticos enfermeros de la NANDA, y finalmente; en la tercera fase, se prescribieron las principales intervenciones de cuidado a implementar tras el egreso de pacientes con EVC.

Discusión y conclusiones: El plan de egreso forma parte del cuidado integral y humanizado, requiere llevarse a cabo de forma habitual para facilitar el diálogo sobre las incertidumbres, dudas y angustias presentes, frecuentemente, en el cuidador primario y enfermo tras su egreso. A su vez, las guías de cuidado contribuyen a establecer el plan de egreso al enfermo, con el propósito de disminuir los reingresos hospitalarios, prevenir complicaciones y mejorar su calidad de vida, además de poder seguir innovando la práctica clínica de enfermería. Palabras clave: Plan de alta; atención domiciliaria de salud; calidad de vida; accidente cerebrovascular; cuidados de enfermería; México.

\section{ABSTRACT}

Introduction: Persons who suffer a stroke generally are not able to carry out their basic activities and thus become dependent. Because of this, designing discharge plans based on wide-scope information facilitating nursing staff provide healthcare education to victims of stroke and their families is of paramount importance.

Methods: In the proposal three stages were considered: a literature review; a main topic delimitation; and a discharge plan, herein featured.

Results: In the first stage, 39 studies were analyzed: 16 qualitative; 11 quantitative; 8 clinical practice guides; 3 mixed methods; and 2 dissertations. In the second stage, the main theme was established based on the scientific criteria and the NANDA nursing diagnostics. In the third stage, the main cerebrovascular disease care interventions were prescribed.

Discussion and conclusions: The integral discharge plan requires being regularly carried out in order to foster dialogues on related uncertainties, doubts, and anxieties. These care guides can contribute to the establishment of discharge plans aimed at reducing hospital readmissions, preventing complications, and improving the quality of life of these patients. Keywords: Discharge plan; home care; quality of life; stroke; nursing care; Mexico.

\section{RESUMO}

Introdução: As pessoas que sofrem um Evento Vascular Cerebral (EVC), geralmente, ficam incapacitadas para a realização de suas atividades básicas, isto, envolve uma dependência, por isso é importante a elaboração de um plano de egresso com vasta informação sobre os cuidados, que facilite no profissional de enfermagem proporcionar educação para a saúde, dirigida a familiares e doentes sobre o cuidado no lar trás um EVC.

Métodos: O procedimento utilizado para esta proposta consistiu três fases, nas quais, se realizou uma revisão da literatura, uma delimitação com base no tema central, para finalmente, elaborar o plano de egresso que aqui se apresenta.

Resultados: Na primeira fase se analisaram 39 estudos, 16 de tipo qualitativo, 11 quantitativos, oito Guias de prática clínica, três mistos e duas teses; posteriormente, na segunda fase se estabeleceu a delimitação do tema com base no critério de evidencia científica e dos diagnósticos enfermeiros da NANDA, e finalmente; na terceira fase, prescreveram-se as principais intervenções de cuidado a implementar trás o egresso de pacientes com EVC.

Discussão e conclusões: O plano de egresso forma parte do cuidado integral e humanizado, requer ser feito de forma habitual, para facilitar o diálogo sobre as incertezas, dúvidas e angústias presentes, frequentemente, no cuidador primário e o doente trás seu egresso. As guias de cuidado contribuem a estabelecer o plano de egresso para o doente, com o propósito, de 
diminuir os reingressos hospitalários, prevenir complicações e melhorar sua qualidade de vida, além de poder seguir inovando a prática clínica de enfermagem.

Palavras chave: Plano de egresso; atenção domiciliar de saúde; qualidade de vida; acidente cerebrovascular; cuidados de enfermagem; México.

\section{INTRODUCCIÓN}

El plan de egreso es un documento que permite a Enfermería ofrecer al cuidador primario, al enfermo y la familia, una amplia información sobre los cuidados que se deben realizar en casa al egreso del paciente de las unidades hospitalarias, con la finalidad de prevenir complicaciones, disminuir reingresos hospitalarios y mejorar la calidad de vida de este ${ }^{1}$.

A nivel mundial, el Evento Vascular Cerebral (EVC) representa la segunda causa de muerte según la $\mathrm{OMS}^{2}$. Cuidar en el hogar a un enfermo con secuelas de EVC, implica cambios en las actividades cotidianas del cuidador primario y familia, que involucran no sólo el cuidado físico, sino también, un cuidado emocional, social y espiritual, pues entre las secuelas provocadas por dicho estado de salud se encuentran alteraciones motoras y sensitivas que le incapacitan en la realización de sus actividades de la vida diaria y provocan en él un alto grado de dependencia.

Enfermería, como agente de cuidado, debe ofrecer una amplia información al enfermo, al cuidador primario y la familia sobre el cuidado que debe llevarse a cabo en el hogar posterior al egreso hospitalario, a través de un plan oportuno y personalizado, que genere confianza y seguridad para la realización correcta y pertinente de cada cuidado en el hogar. Una herramienta útil y práctica puede ser la nemotecnia CUIDARME33, ya que agrupa información suficiente sobre el cuidado integral tras el egreso del paciente a su domicilio, además, resulta de fácil memorización.

El objetivo del presente trabajo es la elaboración de un plan de egreso sustentado en evidencia bibliográfica, dirigido a profesionales de enfermería que tengan bajo su cuidado a pacientes con un alto grado de dependencia a consecuencia de un EVC, con una visión integral para los cuidados en el hogar. Así mismo, tiene la intención de educar a cuidadores, enfermos y familia, en búsqueda de satisfacer necesidades básicas, al igual que prevenir un posible reingreso hospitalario.

\section{MÉTODOS}

Para el presente estudio se llevó a cabo una revisión sistematizada de la literatura, una delimitación con base en el tema central, para finalmente, elaborar un plan de egreso. El procedimiento utilizado en esta propuesta constó de tres fases donde:

- La primera: consistió en la búsqueda y selección de artículos científicos relacionados con el tema tratado, así como la identificación de las principales secuelas tras un EVC.

- La estrategia de búsqueda incluyó artículos de estudios descriptivos, revisiones sistematizadas, meta-análisis y guías de práctica clínica. Las bases de datos usadas fueron: Medline, Scielo, Redalyc, BVS, Bidi UNAM y Cochrane.

- En la segunda: se determinaron los principales diagnósticos de enfermería en el paciente con EVC, según las guías NANDA y la fundamentación bibliográfica.

- La tercera:consistió en establecer los principales cuidados que deben conocer y llevar a cabo los pacientes y cuidadores primarios tras el egreso de una persona con EVC. Se diseñaron tablas de análisis que integran los principales cuidados encontrados, los cuales se organizaron con base en la nemotecnia CUIDARME, que corresponde a: Comunicación efectiva, Urgencias y signos de alarma, Información, Dieta, Ambiente, Recreación y uso del tiempo 
libre, Medicamentos y tratamientos farmacológicos, y Espiritualidad3; debido a su fácil memorización tanto para el profesional de la salud como para el cuidador y enfermo, además de englobar puntos clave para la orientación e información que debe llevar un plan de egreso.

\section{RESULTADOS}

En resultados de la primera fase, se obtuvo un total de 39 estudios analizados, de los cuales, 16 fueron de tipo cualitativo, 11 de tipo cuantitativo, ocho Guías de práctica clínica, tres mixtos y dos tesis. Los estudios cualitativos fueron de tipo descriptivo, exploratorio, de intervención prospectiva y un estudio de caso con observación participante. Los estudios cuantitativos fueron: descriptivos transversales y ensayos controlados aleatorizados.

En el análisis de la información se encontró que la principal afectación que tienen las personas tras un EVC es el deterioro de la movilidad ${ }^{4-6}$, la cual está relacionada con disfunción de orden motora, espasticidad, contracciones o sincinesias, lo que dificulta la ejecución de movimientos voluntarios7. Otra de las principales secuelas generada por esta afección son las deficiencias cognitivas, este tipo de alteración va a depender del hemisferio afectado y de la extensión; sin embargo, cuando se presentan en el hemisferio izquierdo, se pueden producir afasias que alteran el lenguaje, gesto y comunicación. Más aún, las lesiones en el hemisferio derecho pueden generar alteraciones en la percepción u orientación espacial, la conducta emocional, aspectos no verbales de la comunicación como reconocimiento y recuerdo de caras, voces y melodías 8 . Por lo tanto, la comunicación es una de las secuelas con mayor repercusión en la vida del enfermo, pues también se puede ver afectada la deglución y trastornos vesicoesfinterianos ${ }^{6,7}$.

Con base en lo anterior, la inmovilidad es la principal secuela tras un EVC, debido a que se ven afectadas otras necesidades, lo que provoca que el enfermo no pueda realizar, en la mayoría de los casos, sus actividades de la vida diaria, por lo cual depende de un equipo multidisciplinar, un cuidador primario y la familia para la satisfacción de sus necesidades.

El personal de enfermería es un elemento clave para la recuperación de las personas que han padecido un EVC, ya que estos profesionales son quienes brindan cuidados específicos para este tipo de enfermos, además de fungir como educadores para la salud de la persona enferma y cuidadores primarios sobre los tratos y cuidados que se deben realizar una vez que los pacientes se encuentran en su domicilio.

De la delimitación temática, propia de la segunda fase, se encontró que las etiquetas diagnósticas de enfermería más comunes, según la NANDA 2018-20209 y de acuerdo con la revisión bibliográfica para personas con el diagnóstico de EVC, son las presentes en la Tabla 1.

\section{Plan de Egreso. Nemotecnia CUIDARME}

Como resultado de la tercera fase, se obtuvo que entre los principales cuidados que los enfermos, cuidadores primarios y familia deben conocer y llevar a cabo al egreso hospitalario del paciente se encuentran los siguientes:

Comunicación efectiva. Hace referencia a la información y enseñanzas que Enfermería aporta al cuidador primario y enfermo sobre los cuidados a realizar en el hogar tras el egreso hospitalario, esto con la intención de prevenir complicaciones, reingresos hospitalarios, así como mejorar la calidad de vida del afectado. De igual forma, se refiere a la comunicación entre los profesionales de salud, en tanto que puedan compartir la información sobre el tratamiento a seguir, la información relevante y punto de vista de cada profesional. 
Urgencias y signos de alarma. En este punto, el profesional de enfermería debe informar sobre los signos y síntomas de alarma que puede presentar el enfermo, estos pueden indicar un deterioro de la salud o recaída, por lo que resulta necesario, que el cuidador y enfermo los conozcan, además de las acciones que se deben llevar a cabo si se llegasen a presentar (Tabla 2).

Información. El cuidador primario, el enfermo y la familia deben recibir una capacitación suficiente, sobre cómo proporcionar los cuidados específicos y personalizados una vez que el paciente sea dado de alta y se encuentre en su domicilio (Tabla 3).

Dieta. Para este punto se encontró que principalmente se debe orientar al enfermo y cuidador acerca de la higiene de los alimentos, el tipo de alimentos que se pueden consumir, así como su preparación, acorde a la dieta indicada por el nutriólogo (Tabla 4).

Ambiente. Para ofrecer una orientación adecuada, es necesario indagar previamente acerca de las condiciones en las que se encuentra la vivienda y el ambiente laboral (si fuera el caso), para así, ofrecer recomendaciones alcanzables, siempre con el objetivo de mantener la seguridad del enfermo, así como su confort ${ }^{2}$ (Tabla 5).

Recreación y uso de tiempo libre. A través de este punto se sugiere integrar un plan de rehabilitación donde se involucre a la familia para su realización. Esta serie de terapia, además de mejorar la salud del enfermo, ayudará a mejorar su estado de ánimo sin dejar de lado la realización de actividades agradables para él (Tabla 6).

Medicamentos y tratamientos farmacológicos. Para este apartado, la información debe ser clara y suficientemente explícita, donde engloben todos los cuidados que deben efectuarse para cada medicamento, así como las sugerencias para que el tratamiento sea más efectivo. Las principales recomendaciones se encuentran en la Tabla 7.

Espiritualidad. Es de gran importancia tomar en cuenta las recomendaciones acerca de la espiritualidad, pues se busca que el enfermo encuentre un significado de vida para su bienestar ${ }^{42}$.

La espiritualidad para muchas personas se expresa de acuerdo a sus creencias, experiencias, significados de vida, etc., por lo que la intervención que Enfermería lleve a cabo, además de dirigir al enfermo y familia a una terapia de tanatología, es la de acompañamiento, que incluye el contacto visual, auditivo y físico.

Considerar en nuestro plan de egreso este apartado, influirá positivamente en la salud del enfermo y la familia, al afrontar y prevenir la enfermedad, así como disminuir el sufrimiento y dolor ${ }^{42}$.

Tabla 1. Diagnósticos de enfermería

Limpieza ineficaz de las vías aéreas
Riesgo de aspiración ${ }^{10}$
Estreñimiento
Deterioro de la movilidad en cama
Trastorno del patrón del sueño
Déficit de autocuidado: vestido
Deterioro de la integridad cutánea
Riesgo de infección
Riesgo de caídas
Deterioro de la comunicación
Disposición para mejorar el cuidado de familiar
enfermo $^{11}$

Tabla 2. Principales signos y sintomas de alarma en pacientes con secuelas de EVC 9,12,13

Somnolencia por períodos anormales
Dolor persistente de cabeza
Vómito
Convulsiones
Cambios en la visión
Confusión o desorientación
Dificultad para hablar
Dolor intenso que no disminuye con los
medicamentos indicados
Al detectar la presencia de lesiones por presión
en la piel
Al presentar sólo de 2-3 evacuaciones a la
semana
Hipertensión
Broncoaspiración


Tabla 3. Información de los cuidados en el hogar de una persona con dependencia secundaria a EVC

\begin{tabular}{|c|c|c|}
\hline Técnica & Información & Educación para la salud \\
\hline Lavado de manos ${ }^{14}$ & $\begin{array}{l}\text { *Técnica de lavado de } \\
\text { manos, cinco momentos }\end{array}$ & $\begin{array}{l}\text { Enseñanza sobre la técnica de lavado de manos, cinco } \\
\text { momentos y la importancia que tiene para el cuidado } \\
\text { del enfermo en el hogar. }\end{array}$ \\
\hline $\begin{array}{l}\text { Manejo de vía } \\
\text { aérea }\end{array}$ & $\begin{array}{l}{ }^{*} \text { Cuidado de Traqueostomía } \\
{ }^{*} \text { Aspiración de secreciones } \\
{ }^{*} \text { Oxigenoterapia } \\
{ }^{*} \text { Fisioterapia pulmonar } \\
{ }^{*} \text { Identificar datos de } \\
\text { esfuerzo respiratorio }{ }^{4,15}\end{array}$ & $\begin{array}{l}\text { Enseñar sobre la técnica de aspiración de secreciones, } \\
\text { explicando sus posibles complicaciones, el material } \\
\text { requerido, los cuidados del oxígeno en el hogar, } \\
\text { técnicas que favorezcan la expulsión de secreciones, } \\
\text { además de identificar y saber actuar ante datos de } \\
\text { esfuerzo respiratorio. }\end{array}$ \\
\hline $\begin{array}{l}\text { Cuidado de sonda } \\
\text { de gastrostomía }{ }^{16}\end{array}$ & $\begin{array}{l}\text { *Limpieza del estoma y } \\
\text { cuidado de la piel }\end{array}$ & $\begin{array}{l}\text { Educación para la salud respecto al material y } \\
\text { procedimiento sobre la limpieza del estoma y la } \\
\text { prevención de lesiones e infecciones en la piel, } \\
\text { además del cambio de sonda de acuerdo al tipo de } \\
\text { material. }\end{array}$ \\
\hline $\begin{array}{l}\text { Cuidado de la piel e } \\
\text { higiene }^{17,18}\end{array}$ & $\begin{array}{l}\text { *Técnica de baño en cama/ } \\
\text { silla } \\
\text { *Hidratación de la Piel }\end{array}$ & $\begin{array}{l}\text { Enseñanza sobre la técnica del baño de esponja. } \\
\text { Utilización de crema hidratante ouso de aceites } \\
\text { hiperoxigenados. }\end{array}$ \\
\hline $\begin{array}{l}\text { Prevención de } \\
\text { lesiones por } \\
\text { presión }\end{array}$ & $\begin{array}{l}\text { *Prevenir complicaciones } \\
\text { secundarias por la } \\
\text { inmovilización }\end{array}$ & $\begin{array}{l}\text { Capacitación sobre la movilización del enfermo cada } \\
\text { dos horas y las diversas posiciones. } \\
\text { Alineación de segmentos, utilización de almohadas } \\
\text { y colchón de presiones alternas. }\end{array}$ \\
\hline $\begin{array}{l}\text { Prevención de } \\
\text { hombro doloroso } \\
20\end{array}$ & ${ }^{*}$ Movilización de hombro & $\begin{array}{l}\text { Se debe enseñar la movilización del hombro a } 90^{\circ} \text { con } \\
\text { estiramientos suaves, colocación correcta de hombro } \\
\text { pléjico. }\end{array}$ \\
\hline $\begin{array}{l}\text { Prevención de } \\
\text { estreñimiento }^{21}\end{array}$ & ${ }^{*}$ Masaje al marco cólico & $\begin{array}{l}\text { Enseñanza breve sobre la anatomía del colon, } \\
\text { técnica de masaje al marco cólico; especificar } \\
\text { contraindicaciones y recomendaciones ante dicha } \\
\text { técnica. }\end{array}$ \\
\hline $\begin{array}{l}\text { Recomendaciones } \\
\text { para la asistencia } \\
\text { a terapia física, } \\
\text { de lenguaje y } \\
\text { foniatría }\end{array}$ & ${ }^{*}$ Trabajo Multidisciplinar & $\begin{array}{l}\text { Informar sobre la importancia de acudir a terapias de } \\
\text { lenguaje para mejorar la comunicación con el apoyo } \\
\text { de especialistas }{ }^{21} \text {. Uno de los principales problemas } \\
\text { que limitan la comunicación es la dislalia y disfagia } \\
\text { como complicación de un EVC } \mathrm{EV}^{6,7} \text {. }\end{array}$ \\
\hline $\begin{array}{l}\text { Utilización } \\
\text { de tablero de } \\
\text { comunicación }\end{array}$ & & $\begin{array}{l}\text { Enseñar la utilización del tablero de comunicación } \\
\text { y sus beneficios, tanto para el enfermo como para el } \\
\text { cuidador }{ }^{22,23} \text {. }\end{array}$ \\
\hline $\begin{array}{l}\text { Movilización del } \\
\text { enfermo }\end{array}$ & $\begin{array}{l}\text { Mecánica corporal e } \\
\text { higiene de columna }\end{array}$ & $\begin{array}{l}\text { Enseñanza de técnicas de movilización del enfermo, } \\
\text { dirigida al cuidador primario, con la finalidad de } \\
\text { prevenir lesiones musculoesqueléticas y conservar } \\
\text { energía corporal al adoptar posturas ergonómicas }{ }^{18} \text {. }\end{array}$ \\
\hline
\end{tabular}

Conforme a los puntos señalados, es necesario que se promueva en todos los niveles de atención la implementación del plan de egreso desde el ingreso o poco después de éste, asignarle el importante valor que tiene y evaluar en forma constante el costo-efectividad que trae para el sector salud y para el enfermo este tipo de enseñanza.

Por lo anterior, es necesario incrementar en las instituciones lineamientos, protocolos e instrumentos dirigidos al plan de egreso y verificar que se lleven a cabo. Aunado a esto, se debe considerar que un gran reto a superar es la alta demanda de trabajo que el profesional de enfermería enfrenta, lo que influye en no ofrecer al enfermo y cuidador primario una enseñanza adecuada; sin embargo; un apoyo pudiera encontrarse en la participación de especialistas que proporcionen información amplia, específica y personalizada ${ }^{19}$. 
Tabla 4. Recomendaciones para las personas que padecieron un EVC

\begin{tabular}{|c|c|c|}
\hline Técnica & Información & Educación para la salud \\
\hline $\begin{array}{l}\text { Preparación de } \\
\text { alimentos }\end{array}$ & $\begin{array}{l}\text { *Tipos de espesantes } \\
{ }^{*} \text { Alimentos de consistencia blanda } \\
\text { *Evitar texturas mixtas }\end{array}$ & $\begin{array}{l}\text { Enseñanza del tipo de dieta que debe } \\
\text { llevar en casa el paciente, recomendación } \\
\text { de alimentos y dar opciones de menú, } \\
\text { así como espesantes en los alimentos } \\
\text { líquidos y evitar alimentos de texturas } \\
\text { mixtas para prevenir el riesgo de } \\
\text { aspiración }{ }^{24,25} \text {. }\end{array}$ \\
\hline $\begin{array}{l}\text { Recomendaciones } \\
\text { para favorecer la } \\
\text { alimentación }\end{array}$ & $\begin{array}{l}{ }^{*} \text { Alimentar en pequeñas cantidades } \\
{ }^{*} \text { Colocar al paciente en posición de } 90^{\circ} \\
{ }^{*} \text { Vigilar la aparición de síntomas como } \\
\text { tos o disnea durante la alimentación } \\
{ }^{*} \text { Colocar los alimentos en el lado sano de } \\
\text { la boca } \\
{ }^{*} \text { Dar tiempo al enfermo para la } \\
\text { deglución de cada alimento }\end{array}$ & $\begin{array}{l}\text { Orientar a la familia que trasun EVC, } \\
\text { las personas llegan a presentar cierta } \\
\text { limitación de la fuerza y control de } \\
\text { la lengua, por lo que es necesario } \\
\text { realizar cuidados específicos durante la } \\
\text { alimentación; se explica la importancia } \\
\text { de cada uno de estos para la prevención } \\
\text { de complicaciones y mejorar el bienestar } \\
\text { del enfermo y de la propia familia }{ }^{26} \text {. }\end{array}$ \\
\hline $\begin{array}{l}\text { Alimentación } \\
\text { por sonda de } \\
\text { gastrostomía }\end{array}$ & $\begin{array}{l}\text { *Preparación de alimentos } \\
{ }^{*} \text { Velocidad de infusión } \\
{ }^{*} \text { Horarios recomendados } \\
\text { *Temperatura de los } \\
\text { alimentos } \\
{ }^{*} \text { Alimentación en } \\
\text { posición de } 30^{\circ} \text { a } 45^{\circ} \\
{ }^{*} \text { Lavado de la sonda tras } \\
\text { la alimentación y la medicación }{ }^{27 .}\end{array}$ & $\begin{array}{l}\text { Enseñanza sobre los cuidados de la } \\
\text { alimentación con sonda de gastrostomía } \\
\text { en la persona dependiente tras un EVC. } \\
\text { Esde gran importancia explicar de } \\
\text { forma detallada cada cuidado, al igual } \\
\text { que disipar las dudas de los cuidadores } \\
\text { yenfermo con la finalidad de prevenir } \\
\text { complicaciones y disminuir reingresos } \\
\text { hospitalarios. }\end{array}$ \\
\hline $\begin{array}{l}\text { Toma de glicemia } \\
\text { capilar }^{28,29}\end{array}$ & $\begin{array}{l}\text { *Técnica de toma de glicemia capilar } \\
\text { (especificar niveles normales de glucosa) } \\
\text { *Importancia de mantener niveles } \\
\text { normales }\end{array}$ & $\begin{array}{l}\text { Orientación y enseñanza de la técnica } \\
\text { de toma de glicemia capilar, informar } \\
\text { los niveles normales y su importancia en } \\
\text { enfermos que presentaron un EVC y que } \\
\text { son egresados a su domicilio. }\end{array}$ \\
\hline Hidratación ${ }^{30,31}$ & $\begin{array}{l}{ }^{*} \text { Recomendación de la ingesta de } \\
\text { líquidos }\end{array}$ & $\begin{array}{l}\text { Enseñanza de la hidratación del enfermo, } \\
\text { uso de lajarra del buen beber. Posición } \\
\text { correcta del enfermo. }\end{array}$ \\
\hline Higiene bucal & *Técnica de cepillado de dientes & $\begin{array}{l}\text { Enseñanza de la técnica de cepillado de } \\
\text { dientes, su importancia y beneficios }{ }^{32} \text {. }\end{array}$ \\
\hline
\end{tabular}

Tabla 5. Principales recomendaciones del entorno

\begin{tabular}{|c|c|}
\hline Recomendación & Educación para la salud \\
\hline $\begin{array}{l}\text { Higiene del hogar } \\
\text { Apartar objetos que puedan obstruir el paso } \\
\text { Utilizar barreras de apoyo (pasamanos) } \\
\text { Movilidad fuera de cama } \\
\text { Iluminación adecuada } \\
\text { Uso de antiderrapantes } \\
\text { Calzado cómodo } \\
\text { Evitar caminar sobre suelo mojado } \\
\text { Uso de aditamentos o auxiliares de la marcha } \\
\text { (bastón, andadera) } \\
\text { Uso de férulas4 }\end{array}$ & $\begin{array}{l}\text { Enseñanza sobre la importancia de tener un } \\
\text { entorno seguro para el enfermo tras sufrir un } \\
\text { EVC, que permita disminuir el riesgo de caídas } \\
\text { y favorecer la reinserción a sus actividades de la } \\
\text { vida diaria' }\end{array}$ \\
\hline
\end{tabular}


Tabla 6. Recomendaciones sobre uso de tiempo libre y rehabilitación

\begin{tabular}{|c|c|}
\hline Recomendación & Capacitación \\
\hline Ejercicio terapéutico 7,36,37 & $\begin{array}{l}\text { Orientación sobre la asistencia a terapias de rehabilitación. } \\
\text { Realizar en casa } 1 \text { a } 2 \text { veces al día los ejercicios indicados en las } \\
\text { terapias rehabilitadoras (movilizaciones articulares). }\end{array}$ \\
\hline $\begin{array}{l}\text { Aplicación de técnicas de } \\
\text { estimulación sensoperceptivas }\end{array}$ & $\begin{array}{l}\text { Enseñanza de la aplicación de diferentes texturas sobre los } \\
\text { miembros inferiores y superiores del enfermo, para revertir en lo } \\
\text { posible los trastornos sensoriales; orientar sobre que texturas son } \\
\text { las recomendadas y en qué orden se deben aplicar. }\end{array}$ \\
\hline $\begin{array}{l}\text { Mantener estabilidad, alineación } \\
\text { del tronco y articulaciones } \\
\text { proximales y modificar reacciones } \\
\text { posturales }^{38}\end{array}$ & $\begin{array}{l}\text { Enseñanza sobre la técnica correcta de las distintas posiciones en las } \\
\text { que el enfermo se debe encontrar para disminuir complicaciones } \\
\text { como contracturas, subluxaciones, problemas de la piel, entre otras. }\end{array}$ \\
\hline Evitar sobreestimulación 39 & $\begin{array}{l}\text { Orientar y especificar que en el hogar se debe continuar con los } \\
\text { ejercicios realizados en la terapia de rehabilitación y realizar } \\
\text { descansos para no agotar los músculos. }\end{array}$ \\
\hline Participación en grupos de apoyo & $\begin{array}{l}\text { Orientar y fomentar las redes de apoyo en enfermos dependientes } \\
\text { secundario a un EVC, así como las interacciones sociales positivas } \\
\text { que favorezcan el estado de ánimo del paciente y del cuidador } \\
\text { primario }{ }^{40,41} \text {. }\end{array}$ \\
\hline $\begin{array}{l}\text { Realizar paseos o visitas cerca del } \\
\text { domicilio }\end{array}$ & 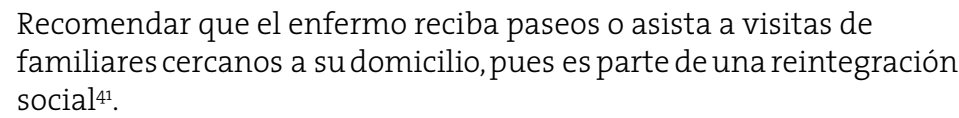 \\
\hline
\end{tabular}

Tabla 7. Orientación ante la medicación

Informar sobre la indicación para cada medicamento

Explicar los posibles efectos adversos

Sugerir los horarios más adecuados para la persona de acuerdo a su ritmo de vida, además de las

características de los medicamentos

Indicar la vía de administración

Informar sobre la importancia de combinar el medicamento con alimentos o alejados de estos

Orientar sobre los riesgos ante la automedicación

Estrategias a seguir ante la polifarmacia (uso de pastilleros, alarmas, etc.)

\section{DISCUSIÓN}

Una de las tareas de gran relevancia para el profesional de enfermería es la elaboración del plan de egreso, donde antes de este, se oriente y capacite al cuidador primario, principalmente en relación a los cuidados que debe llevar a cabo en el hogar.

En este caso se desarrolla el plan para una persona dependiente como consecuencia de un EVC, se utiliza la nemotecnia CUIDARME, ya que emplea puntos clave para la adecuación y organización de la información que se debe enseñar al enfermo y cuidador primario, y en consecuencia, resulta una efectiva estrategia para no pasar por alto ningún elemento básico para el cuidado de la persona. Pinzón ${ }^{3}$ (autor de la nemotecnia), enfatiza que al llevar a cabo el plan de egreso a través de esta herramienta reforzará y ampliará el conocimiento de los cuidadores, lo que favorecerá la seguridad del enfermo y su calidad de vida.

La elaboración y el desarrollo de un plan de egreso no sólo influye en beneficio de los enfermos y sus familias, ya que además de fomentar la participación activa de estos, así como la adherencia 
al tratamiento como lo mencionan Vargas y Fernández ${ }^{43}$, también beneficia al sector salud, al disminuir los reingresos hospitalarios, con lo que se aminoran los costos de la atención.

No obstante, para los enfermos que afrontan una alta dependencia como consecuencia del EVC, es necesario iniciar con la implementación del plan de egreso prácticamente desde su ingreso, pues esto permitirá que el cuidador primario se vaya familiarizando con los cuidados personalizados que requiere su familiar, lo que se relaciona con lo mencionado por Sánchez et al.44, al decir que la/el enfermera(o) debe adelantar dicho plan por escrito desde el ingreso del paciente, o bien, poco después de este, por ello es necesario tener constante comunicación con el médico y otros profesionales de la salud, pues permitirá brindar información clara y precisa en colaboración con otras disciplinas. En adición, se requiere una adecuada valoración tanto del enfermo y su entorno, así como identificar al cuidador primario y determinar la posibilidad de redes de apoyo para evitar la sobrecarga del cuidador ${ }^{45.46}$, para realizarla es necesaria una valoración completa y exhaustiva a fin de establecer un plan de egreso completo, adecuado e individualizado para cada persona.

Por consiguiente, es necesario que se implementen guías y protocolos de cuidado que incluyan la educación al enfermo y cuidador primario durante la hospitalización, orientado hacia el plan de egreso, con la utilización de diversas estrategias de enseñanza como las señaladas por Bjartmarz et al.4, ya que es necesario preparar al enfermo y cuidador primario, dotándolos con un amplio conocimiento durante su estancia hospitalaria. Así mismo, la enseñanza debe ser repetitiva y constante para que la información quede clara y se lleven a cabo la intervenciones necesarias de forma efectiva en el hogar; por lo que es importante ofrecer seguimiento tras el egreso para verificar el bienestar del enfermo.

\section{CONCLUSIÓN}

El EVC representa una enfermedad incapacitante para quien la padece, esto resulta un gran reto para los profesionales de la salud, sin embargo; para que la recuperación del enfermo sea lo más temprana posible con efectos positivos en la calidad de vida del enfermo y la familia, es necesario la atención oportuna y de calidad del profesional de enfermería, pues es enfermería quien ofrece cuidados las 24 horas del día.

El plan de egreso es una herramienta útil y de gran relevancia para el enfermo, el cuidador primario y la familia pues, a través de éste, se fomenta la disminución de reingresos hospitalarios por complicaciones, mejora la calidad de vida de los enfermos y familia, además de disminuir los costos de los servicios de atención médica en el sector salud.

Es necesario tomar en consideración que el plan de egreso, al aumentar la preparación de cuidadores y la satisfacción de sus necesidades, mejora su calidad de vida, del mismo modo, fortalece la conciencia de estos para mejorar su salud y modificar conductas poco saludables.

Sin duda alguna, el plan de egreso también llamado plan de alta, es una intervención de Enfermería que forma parte del cuidado integral y humanizado, mismo que debe de llevarse a cabo de forma habitual, con el fin de facilitar el diálogo sobre las incertidumbres, dudas y angustias que se presentan, frecuentemente, en el cuidador primario y el enfermo tras su egreso.

En diferentes partes del mundo ya se están implementando las consejerías de enfermería presencial y telefónica, lo que es una fuente útil e innovadora para la práctica clínica, que tiene como resultados la disminución de reingresos hospitalarios a consecuencia de un mejor cuidado en el hogar de los enfermos tras recibir una intervención educativa en la consulta de enfermería dirigida tanto 
a pacientes como a familiares. De igual forma, la consejería de enfermería está basada en evidencia científica, siempre tomando en consideración un acompañamiento respetuoso dirigido hacia las respuestas humanas y estado de salud del enfermo y la familia.

Por lo tanto, la/el enfermera(o) como profesional, debe reforzar el pensamiento crítico, desarrollar habilidades de comunicación en términos de solidaridad y escucha con un lenguaje claro y preciso, crear un ambiente de confianza, para así identificar las dudas, temores y expectativas relacionadas con el cuidado y salud del enfermo. Finalmente, tiene que tomar en cuenta las costumbres y valores de cada persona, así como la actualización constante sobre la enseñanza de los cuidados específicos y personalizados que el enfermo y cuidador primario deben conocer y así poder seguir innovando la práctica clínica de enfermería.

\section{RESPONSABILIDADES ÉTICAS}

Protección de personas y animales. Los autores declaran que para este estudio no se han realizado experimentos en seres humanos ni en animales.

Confidencialidad de los datos.Se solicitó consentimiento informado para la protección de los intereses de la persona en cuestión.

Financiamiento. Ninguno.

Conflicto de intereses. En este estudio se declara que no existe conflicto de intereses.

\section{REFERENCIAS}

1. Tixtha LE, Alba LA, Córdoba ÁMÁ, Campos CEM. El plan de alta de enfermería y su impacto en la disminución de reingresos hospitalarios. Rev. enf. neurol. 2014; 13(1): 12-8. https://bit.ly/2Z7yeZc

2. Organización Mundial de la Salud. Las 10 principales causas de defunción. Ginebra: OMS; 2015. https://bit.ly/1nW3SMo

3. Pinzón-de Salazar L. El plan de alta: una herramienta para el cuidado integral y la recuperación de la persona enferma. Hacia Promoc. Salud. 2005; 10(enero-diciembre): 22-7. https://bit.ly/2IY3iP6

4. Bjartmarz I, Jónsdóttir H, Hafsteinsdóttir TB. Implementation and feasibility of the stroke nursing guideline in the care of patients with stroke: a mixed methods study. BMC Nurs. 2017; 16(72): 1-17. https://doi.org/10.1186/s12912-017-0262-y

5. Vives-Medina OT, Quintana-Pereda R, Soto-Páez N. Protocolo de actuación de Enfermería para pacientes con enfermedad cerebrovascular. Rev Ciencias Médicas. 2014; 18(3): 414-29.

https://bit.ly/2OWOyI5

6. Jones SP, Miller C, Gibson JME, Cook J, Price C, Watkins CL. The impact of education and training interventions for nurses and other health care staff involved in the delivery of stroke care: An integrative review. Nurse Educ Today. 2018; (61): 249-57. https://doi.org/10.1016/j.nedt.2017.11.024

7. Devesa-Gutiérrez I, Mazadiego-González ME, Hernández-Hernández MÁB, Mancera-Cruz HA. Rehabilitación del paciente con enfermedad vascular cerebral (EVC). Rev. Mex. Med. Fís. y Rehab. 2014; 26(3-4): 94-108. https://bit.ly/2Mmi7AG

8. Ardila-Rodríguez WA, Silva-Sieger FA, Acosta-Barreto MR. Perfil neuropsicológico en pacientes con ACV isquémico de la arteria cerebral media izquierda. Act Neu Col. 2013; 29(1): 36-43. https://bit.ly/2MmmliD

9. NANDA International. Diagnósticos enfermeros definiciones y clasificación 2018-2020.11 a ed. Barcelona: Elsevier; 2018. 
10. Oliveira ARS, Costa AGS, Morais HCC, Cavalcante TF, Lopes MVO, Araujo TL. Factores clínicos predictores del riesgo para aspiración y aspiración respiratoria en pacientes con accidente cerebro vascular. Rev. Lat.-Am. Enferm. 2015; 23(2): 216-24. https://doi.org/10.159o/0104-1169.0197.2545

11. Instituto Aragonés de Ciencias de la Salud. Guía de Práctica Clínica para el manejo de pacientes con Ictus en Atención Primaria. Aragón, España: Gobierno de España; 2009. https://bit.ly/2HgIq7n

12. Ameriso SF, Reynoso F. Aumentando la prevención y el tratamiento del accidente cerebrovascular en el continente americano: Declaración de Santiago de Chile, 31 de octubre del 2015. Neurolog. argent. 2016; 8(2):71-3. https://doi.org/10.1016/j.neuarg.2016.03.005

13. Secretaría de Salud. Guía de Práctica Clínica. Prevención secundaria, diagnóstico, tratamiento y vigilancia de la enfermedad vascular cerebral isquémica. Ciudad de México: CENETEC; 2018. https://bit.ly/1DEnL6w

14. Bierhals CCBK, Santos NO, Fengler FL, Raubustt KD, Forbes DA, Paskulin LMG. Necesidades de los cuidadores familiares en la atención domiciliaria a ancianos. Rev. Lat.-Am. Enferm. 2017; 25(e2870): 1-8. https://doi.org/10.1590/1518-8345.1511.2870

15. Alonzo C, Ameriso S, Atallah AM, Cirio JJ, Zurrú MC. Consenso de diagnóstico y tratamiento agudo del accidente cerebrovascular isquémico. Rev. argent. cardiol. 2012; 80(5):1-18. http://dx.doi.org/10.7775/rac.es.v80.i5.1596

16. Jaafar MH, Mahadeva S, Tan KM, Ai-Vyrn Chin, Bahyah KS, Hui-Min K. Long-term nasogastric versus percutaneous endoscopic gastrostomy tube feeding in older asians with dysphagia: A pragmatic study. Nutr Clin Pract. 2018; 34(2): 280-9. https://doi.org/10.1002/ncp.10195

17. Cometto MC. Manejo de enfermería en el accidente cerebro vascular inicial. Enfermería glob. 2005; (7): 1-16. https://bit.ly/2H2SaOy

18. González-Consuegra RV, Pérez-Valderrama DC, Valbuena-Flor LF. Prevención de lesiones de piel: educación en el equipo de salud y familiares de personas hospitalizadas. Rev. fac. med. 2016; 64(2): 229-38. https://doi.org/10.15446/revfacmed.v64n2.49903

19. Luque-Moreno C, Peña-Salinas M, Rodríguez-Pappalardo F, López-Rodríguez L. Prevención de úlceras por presión y lesiones musculoesqueléticas: paciente con ictus. Gerokomos. 2012; 23(1): 42-6. https://bit.ly/3ognHrK

20. Palazón-García R, Alonso-Ruíz MT, Martín-Márquez J, Berrocal-Sánchez I. Hombro doloroso en el hemipléjico. Rehabilitación (Madr.). 2014; 38(3):104-7.https://doi.org/10.1016/So048-7120(04)73440-4

21. Arias-Cuadrado Á. Rehabilitación del ACV: evaluación, pronóstico y tratamiento. Galicia clin. 2009; 70(3): 25-40. https://bit.ly/2z9BwfH

22. Gutiérrez-Ruíz K, De los Reyes-Aragón C, Rodríguez-Día M, Sánchez-Herrera A. Técnicas de rehabilitación, neuropsicológica en daño cerebral adquirido: ayudas de memoria externas y recuperación espaciada. Psicol. Caribe. 2009; (24): 147-79. https://bit.ly/2HxDsC8

23. Echavárri-Pérez C. Alteraciones de la comunicación y del lenguaje en la lesión cerebral: afasia. Rehabilitación (Madr.). 2000; 34(6): 483-91. https://bit.ly/2P4tv6G

24. Secretaría de Salud. Guía de Práctica Clínica. Diagnóstico y manejo de la parálisis de Bell (parálisis facial idiopática). Ciudad de México: CENETEC; 2008. https://bit.ly/2EPtRbW

25. Centro de referencia estatal de atención al daño cerebral (CEADAC). Guía de nutrición de personas con disfagia. España: IMSERSO; 2017. https://bit.ly/2BTwToZ

26. Barbié-Rubiera A, Marcos-Plasencia LM, Aguilera-Martínez Y. Disfagia en paciente con enfermedad cerebrovascular. Medisur. 2013; 7(1): 36-44. https://bit.ly/2z85YXN 
27. Miguel-Ruiz E. Cuidados de enfermería en el manejo de la gastrostomía endoscópica percutánea. Una revisión bibliográfica. Valladolid: Universidad de Valladolid; 2018. https://bit.ly/31Xixl5

28. Wainsztein NA, Pujol-Lereis VA, Capparelli FJ, Hlavnika A, Díaz MF, Leiguarda RE, et al. Control moderado de hiperglucemia luego de infarto cerebral agudo en unidad de cuidados intensivos. Medicina (B. Aires). 2014; 74(1): 37-41. https://bit.ly/2TPBYJH

29. Asociación Nacional de Enfermeras de Ontario/Fundación del Corazón de Ontario. Guía de buenas prácticas en enfermería. Valoración del ictus mediante la atención continuada. Toronto, Cánada: RNAO; 2011. https://bit.ly/2H18SE7

30. Bahouth MN, Gottesman RF, Szanton SL. Primary 'Dehydration' and acute stroke: a systematic research review. J. Neurol. 2018; 1432-59. https://doi.org/10.1007/so0415-018-8799-6

31. Lin J, Weng Y, Li M, Mo J, Zhao J. Hydration prevents chronic hyperglycaemic patients from neurological deterioration post-ischaemic stroke. Acta Neurol Scand. 2018; 137(6): 557-65.

https://doi.org/10.1111/ane.12900

32. Malik NA,Mohamad YS, Tao LO. Effectivesness of a Web-Based Health Education Program to Promote Oral Hygiene Care Among Stroke Survivors: Randomized Controlled Trial.J Med Internet Res. 2017; 19(3): e87. https://doi.org/10.2196/jmir.7024

33. Pérez RJ, Torres AL. La discapacidad por enfermedad vascular cerebral. Rev Med Inst Mex Seguro Soc. 2012; 50 (3): 249-54. https://cutt.ly/Vwnr5DS

34. Teixeira CP, Silva LD. Las incapacidades físicas de pacientes con accidente vascular cerebral: acciones de enfermería. Enferm. Glob. 2009; (15). https://bit.ly/2HsXuQDD

35. Moyano VA. El accidente cerebrovascular desde la mirada del rehabilitador. Rev Hosp Clín Univ Chile. 2010; 21: 348-55. https://bit.ly/2qzmpZ1

36. Hernández JB, Benjumeca P, Tuso L. Indicadores del desempeño clínico fisioterapéutico en el manejo hospitalario temprano del accidente cerebrovascular (ACV). Rev. Cienc Salud. 2013; 11(1): 7-34 https://bit.ly/2ELHOCJ

37. Rodríguez ML, Díaz CR. Beneficios del ejercicio físico terapéutico en pacientes con secuelas por enfermedad cerebrovascular. Rev cubana med. 2012; 51(3): 258-66. https://bit.ly/2qAhdDx

38. Castro MK, Pérez PM, Moscoso AF, Tanaka C. Transferencia del aprendizaje motor en pacientes con antecedentes de accidente cerebrovascular: serie de casos. Rev. Fac. Med. 2015; 63 (2): 315-20. https://bit.ly/2HAtgc1

39. Consejo de Salubridad General. Guía de Práctica Clínica en Intervenciones de Rehabilitación temprana en el Adulto Mayor Hospitalizado. Ciudad de México: CENETEC 2013. https://bit.ly/1LOH4Ml

40. Doussoulin A, Najum J, Saiz JL, Molina F. Impacto de la rehabilitación neurológica a través de la terapia de restricción inducida modificada en la mejora del apoyo social en usuarios con ataque cerebro vascular. Rev. Chil. Neuro-psiquiatr. 2016; 54(3):187-97.

http://dx.doi.org/10.4067/S0717-92272016000300003

41. Mangini BS, Angelo M. Entre la libertad y la reclusión: el apoyo social como un componente de la calidad de vida del binomio cuidador familiar y persona dependiente. Rev. Latino-Am. Enfermagem. 2008; 16(1): 15-23. http://dx.doi.org/10.1590/S0104-11692008000100003

42. Higuero AM. Percepción y experiencia enfermera sobre atención de la dimensión espiritual de los pacientes en la práctica clínica. [Tesis]. España: Universidad Internacional de Catalunya. Facultad de Medicina y Ciencias de la Salud; 2013.

43. Vargas BZ, Fernández CR. Programa de alta hospitalaria programada para preparar el egreso del paciente y familia. Rev. Enf. 2011; 21: 1-20. https://doi.org/10.15517/REVENF.VoI21.3658 
44. Sánchez B, Carrillo GM, Barrera L. El plan de transición y egreso hospitalario y su efecto en el cuidado de la salud: una revisión integrada. rev.udcaactual.divulg.cient. 2014; 17 (1): 13-23.

http://bit.ly/2HKyTUP

45. Yonte HF, Urión PZ, Martín GM, Montero HR. Sobrecarga del cuidador principal. Revista enfermería CyL. 2010; 2(1): 59-69. https://bit.ly/2HRjuCn

46. Prieto-Miranda SE, Arias-Ponce N, Villanueva-Muñoz EY, Jiménez-Bernardino CA. Síndrome de sobrecarga del cuidador en familiares de pacientes geriátricos atendidos en un hospital de segundo nivel. Med Int Méx. 2015; 31:66o-8. https://cutt.ly/Uwnthkj 